\title{
Disturbance Observer-Based Adaptive Control of Hypersonic Vehicles with Constrained Actuators
}

\author{
Guan Wang $\mathbb{D}^{1},{ }^{1} \mathrm{Li}$ Li $\mathbb{D}{ }^{2}$ Weihua $\mathrm{Li}^{3}{ }^{3}$ Huajun $\mathrm{Zhou}^{3}{ }^{3}$ Changbo $\mathrm{Ma},{ }^{1}$ and Hongwei Xia ${ }^{1}$ \\ ${ }^{1}$ Space Control and Inertial Technology Research Center, Harbin Institute of Technology, Harbin 150001, China \\ ${ }^{2}$ School of New Energy, Harbin Institute of Technology (Weihai), Weihai 264209, China \\ ${ }^{3}$ Shanghai Aerospace Control Technology Institute, Shanghai 201109, China
}

Correspondence should be addressed to Li Li; lili2017@hit.edu.cn

Received 5 August 2020; Revised 28 August 2020; Accepted 13 September 2020; Published 10 October 2020

Academic Editor: Wu Zhonghua

Copyright (c) 2020 Guan Wang et al. This is an open access article distributed under the Creative Commons Attribution License, which permits unrestricted use, distribution, and reproduction in any medium, provided the original work is properly cited.

This study investigates an adaptive controller for the flexible air-breathing hypersonic vehicles (AHVs) subject to external disturbances and actuator constraints. The combination of nonlinear disturbance observer and adaptive mechanism is exploited to design an adaptive controller for each subsystem. For the velocity subsystem, an auxiliary system is employed to handle the scramjet input saturation issue. For the altitude subsystem, the magnitude/rate constraints and the dynamics of aerodynamic control surfaces are addressed by the control allocation module. Simulations show the effectiveness of the proposed control.

\section{Introduction}

Air-breathing hypersonic vehicles (AHVs) have attracted considerable attention in both military and civil fields. Though AHVs have been developed for more than half a century, various studies have shown that some critical technologies, such as thermal protection, high-fidelity modeling, and robust control, still need to be further investigated.

Based on the control-oriented model developed by Parker et al. [1], numerous theoretical studies on the control of AHVs have sprung up [2]. Early research interests focus on state feedback linearization or nonlinear dynamic inversebased control of AHVs [3]. In recent years, the adaptive control has always been a research hotspot in the field of AHV control, and one can refer to the robust adaptive control in $[4,5]$, the sliding mode adaptive control in [6], the trajectory restricted adaptive control in [7], the fault-tolerant adaptive control in $[8,9]$, and the neural adaptive control in $[10,11]$.

Nevertheless, a practical problem in AHV control is the physically limited actuators [12, 13]. Recently, some outstanding works have come out focusing on the input saturation problem of AHVs [14, 15]. [16] used the disturbance observer-based (DOB) feedback linearization control to overcome constraints on actuators. A combination of backstepping design and nonlinear disturbance observer (NDO) is utilized to handle actuator constraints [17]. By introducing the sliding-mode differentiator techniques and the auxiliary systems, [11] developed the distributed finite time faulttolerant containment control to handle the input saturation. On the other side, control allocation (CA) also plays a good role in dealing with the actuator constraints [18]. For instance, [19] handled the saturation using a robust model predictive control algorithm. [20] developed a control allocation scheme for fault-tolerant control based on a linear parameter varying (LPV) system. But the employed LPV model was complex and time-demanding for the CA problem.

Motivated by the above investigations, this paper proposes a disturbance observer-based adaptive control AHVs with constrained actuators. In the velocity loop, the auxiliary system developed in [12] is employed to handle the saturation issue of the scramjet input. In the altitude loop, a control allocation module is exploited for the magnitude/rate constraints and the dynamics of aerodynamic control surfaces. The main contributions of this paper can be briefly outlined as follows: 
(1) The saturation problem of the scramjet, as well as the dynamics and magnitude/rate constraints of the control surface, is taken into consideration

(2) NDO is developed in each step to further improve the disturbance rejection property. The observation errors are suppressed by adaptive control laws

The rest of this paper is organized as follows: Section 2 formulates the considered problem; Section 3 presents the design process; Sections 4 and 5 give a stability analysis and a simulation study, respectively; Section 6 concludes this study.

\section{Problem Statement and Preliminaries}

2.1. Control-Oriented AHV Model. In this paper, we consider the longitudinal control-oriented AHV model developed by Parker et al. [1]:

$$
\begin{gathered}
\dot{V}=\frac{T \cos \alpha-D}{m}-g \sin \gamma+d_{V}, \\
\dot{h}=V \sin \gamma, \\
\dot{\gamma}=\frac{L+T \sin \alpha}{m V}-\frac{g \cos \gamma}{V}+d_{\gamma}, \\
\dot{\alpha}=Q-\dot{\gamma}, \\
\dot{Q}=\frac{M}{I_{y y}}+\sum_{i=1}^{2} \frac{\psi_{i} \ddot{\eta}_{i}}{I_{y y}}+d_{Q}, \\
\ddot{\eta}_{i}=-2 \xi_{i} \omega_{i} \dot{\eta}_{i}-\omega_{i}^{2} \eta_{i}+N_{i}+\psi_{i}^{\prime} \dot{Q}, \quad i=1,2 .
\end{gathered}
$$

In (1)-(5), velocity $V$, altitude $h$, flight path angle (FPA) $\gamma$ , angle of attack (AoA) $\alpha$, and pitch rate (PR) $Q$ are system states; $g$ is the acceleration due to gravity; mass $m$ and moment of inertia $I_{y y}$ are decided by the design and fuel level of AHVs; the disturbances $d_{V}, d_{\gamma}$, and $d_{Q}$ are external disturbances with bounded derivatives; $\eta_{i}$ is the $i$ th generalized elastic coordinate; $\omega_{i}$ and $\xi_{i}$ are the natural frequency and the damping ratio for flexible mode $\eta_{i}$, respectively; $\psi_{i}$ and $\psi_{i}^{\prime}$ are coupling coefficients between rigid-body and flexible dynamics; thrust $T$, lift $L$, drag $D$, pitching moment $M$, and $i$ th generalized force $N_{i}$ have the following curve-fitted approximations:

$$
\begin{aligned}
T= & \bar{q} S\left(C_{T, \Phi}^{3} \alpha^{3}+C_{T, \Phi}^{2} \alpha^{2}+C_{T, \Phi}^{1} \alpha+C_{T, \Phi}^{0}\right) \Phi \\
& +\bar{q} S\left(C_{T}^{3} \alpha^{3}+C_{T}^{2} \alpha^{2}+C_{T}^{1} \alpha+C_{T}^{0}\right), \\
L= & \bar{q} S\left(C_{L}^{1} \alpha+C_{L}^{0}+C_{L}^{\delta_{e}} \delta_{e}+C_{L}^{\delta_{c}} \delta_{c}\right),
\end{aligned}
$$

$$
D=\bar{q} S\left(C_{D}^{2} \alpha^{2}+C_{D}^{1} \alpha+C_{D}^{0}+C_{D}^{\delta_{e}^{2}} \delta_{e}^{2}+C_{D}^{\delta_{e}} \delta_{e}+C_{D}^{\delta_{c}^{2}} \delta_{c}^{2}+C_{D}^{\delta_{c}} \delta_{c}\right),
$$

$$
M=z_{T} T+\bar{q} \bar{c} S\left(C_{M}^{2} \alpha^{2}+C_{M}^{1} \alpha+C_{M}^{0}+C_{M}^{\delta_{e}} \delta_{e}+C_{M}^{\delta_{c}} \delta_{c}\right),
$$

$$
\begin{gathered}
N_{1}=C_{N_{1}}^{2} \alpha^{2}+C_{N_{i}}^{1} \alpha+C_{N_{0}}^{0}, \\
N_{2}=C_{N_{2}}^{2} \alpha^{2}+C_{N_{2}}^{1} \alpha+C_{N_{2}}^{0}+C_{N_{2}}^{\delta_{e}} \delta_{e}+C_{N_{2}}^{\delta_{c}} \delta_{c} .
\end{gathered}
$$

In (7)-(10), scramjet fuel equivalency ratio (FER) $\Phi$, canard deflection angle $(\mathrm{CDA}) \delta_{c}$, and elevator deflection angle (EDA) $\delta_{e}$ are three system inputs, respectively; $\bar{q}$ is the dynamic pressure; $S$ is the reference area; $z_{T}$ is the thrust-to-moment coupling coefficient; $\bar{c}$ is the mean aerodynamic chord; $C_{*}^{*}$ denotes the fitting coefficients of force and moment.

2.2. Problem Formulation. From a practical viewpoint, FER should comply with the saturation property:

$$
\Phi=\operatorname{Sat}\left(\Phi_{c}\right)= \begin{cases}\Phi_{\text {cooling }}, & \Phi_{c}<\Phi_{\text {cooling }} \\ \Phi_{\text {choking }}, & \Phi_{c}>\Phi_{\text {choking }} \\ \Phi_{c}, & \text { otherwise }\end{cases}
$$

where $\Phi_{c}$ is the FER command; $\Phi_{\text {cooling }}$ is the constant lower threshold to keep sufficient heat dissipation through active cooling; the upper bound $\Phi_{\text {choking }}$ is required to prevent the scramjet from thermal choking [12].

In engineering, the magnitude and rate constraints of the actuators (canard/elevator) have a great influence on control performances.

$$
\begin{aligned}
& \mathscr{M}_{L} \leq \delta \leq \mathscr{M}_{U}, \\
& \mathscr{R}_{L} \leq \dot{\delta} \leq \mathscr{R}_{U},
\end{aligned}
$$

where $\delta$ denote EDA $\delta_{c}$ or CDA $\delta_{e} ; \mathscr{M}_{L}<0$ and $\mathscr{M}_{U}>0$ are lower and upper bounds of the magnitude; $\mathscr{R}_{L}<0$ and $\mathscr{R}_{U}$ $>0$ are the corresponding rate bounds. It should be pointed out that the upper and lower amplitude limits of a control surface can be asymmetric in reality, that is, the more common case $\left|\mathscr{M}_{L}\right| \neq \mathscr{M}_{U}$ or $\left|\mathscr{R}_{L}\right| \neq \mathscr{R}_{U}$ is also taken into consideration.

At the end of this subsection, several lemmas crucial for the following design are given.

Lemma 1 (see [21]). $|x| \leq x \tanh (c x / \varepsilon)+\varepsilon$, where $x \in \mathbb{R}, \varepsilon$ $\in \mathbb{R}_{+}$, and $c \approx 0.2785$.

Lemma 2 (see [22]). If a function $W$ satisfies $\dot{W} \leq-a W+b$ with $a \in \mathbb{R}_{+}, b \in \mathbb{R}_{+}$, it has

$$
W(t) \leq \frac{b}{a}+\left[W(0)-\frac{b}{a}\right] e^{-a t}
$$

Lemma 3 (see [23]). Consider a scalar nonlinear system

$$
\dot{x}=H(x)+G(x) u+d,
$$

where $x \in \mathbb{R}$ and $u \in \mathbb{R}$ are system state and control input, respectively; $H(x)$ and $G(x)$ are known scalar functions with 
respect to $x . d \in \mathbb{R}$ represents the disturbance and $|\dot{d}| \leq M_{d}$, where $M_{d}$ is an unknown positive constant.

NDO is constructed as

$$
\left\{\begin{array}{l}
\dot{p}=-l(x)[\lambda(x)+p+H(x)+G(x) u] \\
l(x)=\frac{\partial \lambda(x)}{\partial x} \\
\widehat{d}=p+\lambda(x)
\end{array}\right.
$$

where $\hat{d} \in \mathbb{R}$ is the estimation of $d ; \lambda(x)=L_{d} x$ with $L_{d}>0 ; l($ $x)=\partial \lambda(x) / \partial x=L_{d}$.

Defining the estimation error $\tilde{d}=d-\widehat{d}, \tilde{d}$ is governed by

$$
\dot{\tilde{d}}=\dot{d}-\dot{p}-\frac{\partial \lambda(x)}{\partial x} \dot{x}=-l(x) \tilde{d}+\dot{d}
$$

The estimation error system (18) is bounded-input-bounded output stability, that is, $\tilde{d}$ is bounded with bounded $\dot{d}$.

\section{Controller Design}

3.1. Velocity Tracking Control Design. This section presents an adaptive tracking controller for velocity subsystem (1) ensuring $V \rightarrow V_{\text {ref }}$. The velocity dynamics is first expressed in the following form:

$$
\dot{V}=H_{V}+G_{V} \Phi+D_{V}
$$

where

$$
\begin{aligned}
H_{V}= & \frac{\bar{q} S}{m}\left[\left(C_{T, \Phi}^{3} \alpha^{3}+C_{T, \Phi}^{2} \alpha^{2}+C_{T, \Phi}^{1} \alpha+C_{T, \Phi}^{0}\right) \cos \alpha\right. \\
& \left.-\left(C_{D}^{2} \alpha^{2}+C_{D}^{1} \alpha+C_{D}^{0}\right)\right]-g \sin \gamma \\
G_{V}= & \left(C_{T, \Phi}^{3} \alpha^{3}+C_{T, \Phi}^{2} \alpha^{2}+C_{T, \Phi}^{1} \alpha+C_{T, \Phi}^{0}\right) \cos \alpha \\
D_{V}= & -\bar{q} S\left(C_{D}^{\delta_{e}^{2}} \delta_{e}^{2}+C_{D}^{\delta_{e}} \delta_{e}+C_{D}^{\delta_{c}^{2}} \delta_{c}^{2}+C_{D}^{\delta_{c}} \delta_{c}\right)+d_{V} .
\end{aligned}
$$

First, similar to [23], we introduce a modified tracking error to handle the saturated $\Phi$ as

$$
e_{V}=V-V_{\text {ref }}-\chi_{V}
$$

where $\chi_{V}$ is governed by

$$
\dot{\chi}_{V}=-k_{V} \chi_{V}+G_{V}\left(\Phi-\Phi_{V}\right) \text {, }
$$

with $k_{V}>0$. Then, $\dot{e}_{V}$ is calculated as

$$
\dot{e}_{V}=k_{V} \chi_{V}+H_{V}+G_{V} \Phi+D_{V}-\dot{V}_{\text {ref }} .
$$

Taking into account the bounded $\dot{d}_{V}$ and the finite deflecting velocity of physical control surfaces, $\dot{D}_{V}$ is assumed to be bounded. Hence, the NDO for $D_{V}$ is constructed.

$$
\left\{\begin{array}{l}
\dot{p}_{V}=-l_{V}\left[\lambda_{V}(V)+p_{V}+H_{V}+G_{V} \Phi_{c}\right] \\
l_{V}=\frac{\partial \lambda_{V}(V)}{\partial V} \\
\widehat{D}_{V}=p_{V}+\lambda_{V}(V) .
\end{array}\right.
$$

Defining estimation error as $\tilde{D}_{V}=D_{V}-\widehat{D}_{V}$, it has

$$
\dot{\tilde{D}}_{V}=-l_{V} \tilde{D}_{V}+\dot{D}_{V}
$$

For the fact that $\dot{D}_{V}$ is bounded, $\tilde{D}_{V}$ is also bounded from Lemma 3, i.e., $\left|\tilde{D}_{V}\right| \leq \Theta_{V}$ with $\Theta_{V}>0$.

We design the control law

$\Phi_{c}=\frac{1}{G_{V}}\left[-k_{V}\left(V-V_{\text {ref }}\right)-H_{V}+\dot{V}_{\text {ref }}-D_{V}-\widehat{\Theta}_{V} \tanh \left(\frac{c e_{V}}{\varepsilon_{V}}\right)\right]$,

where $\widehat{D}_{V}$ is estimated by NDO and $\widehat{\Theta}_{V}$ is the estimate of $\Theta_{V}$ , generated by the adaptive law:

$$
\dot{\widehat{\Theta}}_{V}=\sigma_{V}\left[-k_{V}^{\prime} \widehat{\Theta}_{V}-e_{V} \tanh \left(\frac{c e_{V}}{\varepsilon_{V}}\right)\right] \text {, }
$$

where $\sigma_{V}>0, k_{V}^{\prime}>0$, and $\varepsilon_{V}>0$.

3.2. Altitude Tracking Control Design. In the altitude subsystem, control allocation is employed as an attractive technology to handle both canard and elevator servomechanism dynamics and constraints. For convenience, $\delta_{c}^{0}$ and $\delta_{e}^{0}$ represent the desired control command to be designed, $\delta_{c}^{\text {com }}$ and $\delta_{e}^{\text {com }}$ denote the control command after the process of control allocation, and $\delta_{c}$ and $\delta_{e}$ are the real control inputs applied to AHVs with the dynamics $\delta_{c} / \delta_{c}^{\mathrm{com}}=20 /(s+20)$ and $\delta_{e} / \delta_{e}^{\mathrm{com}}$ $=20 /(s+20) \quad[24]$. We also define $\left[\Delta \delta_{c}, \Delta \delta_{e}\right]^{\mathrm{T}}=$ $\left[\delta_{c}-\delta_{c}^{0}, \delta_{e}-\delta_{e}^{0}\right]^{\mathrm{T}}$ for the following design.

3.2.1. Design for $(h, \gamma)$-Dynamics. Recalling the kinematical equation (2), the realization of altitude reference tracking can be transferred to the FPA tracking problem. In this paper, to achieve $h \rightarrow h_{\text {ref }}$, the control system steers the AHVs to track the FPA reference:

$$
\gamma_{\mathrm{ref}}=\arcsin \left[\frac{-k_{V}\left(h-h_{\mathrm{ref}}\right)+\dot{h}_{\mathrm{ref}}}{V}\right]
$$

where $h_{\text {ref }}$ is the smooth altitude reference generated by the traditional second-order command filter; $k_{h}>0$.

Define a time-related function as $\alpha_{f}$, which decides the final AoA. The FPA dynamics can be calculated as

$$
\dot{\gamma}=R_{\Gamma}\left(\alpha-\alpha_{f}\right)+H_{\gamma}+G_{\gamma} \delta_{c}^{0}+D_{\gamma}
$$


where

$$
\begin{aligned}
R_{\Gamma}= & \frac{\bar{q} S}{m V}\left[C_{L}^{1}+\left(C_{T, \Phi}^{3} \alpha^{3}+C_{T, \Phi}^{2} \alpha^{2}+C_{T, \Phi}^{1} \alpha+C_{T, \Phi}^{0}\right) \Phi\right. \\
& \left.+C_{T}^{3} \alpha_{f}^{3}+C_{T}^{2} \alpha_{f}^{2}+C_{T}^{1} \alpha_{f}+C_{T}^{0}\right] \\
H_{\gamma}= & \frac{\bar{q} S}{m V}\left[C_{L}^{1}+\left(C_{T, \Phi}^{3} \alpha_{f}^{3}+C_{T, \Phi}^{2} \alpha_{f}^{2}+C_{T, \Phi}^{1} \alpha_{f}+C_{T, \Phi}^{0}\right) \Phi\right. \\
& \left.+C_{T}^{3} \alpha_{f}^{3}+C_{T}^{2} \alpha_{f}^{2}+C_{T}^{1} \alpha_{f}+C_{T}^{0}\right] \alpha_{f}+\frac{\bar{q} S}{m V} C_{L}^{0}-\frac{g \cos \gamma}{V}, \\
G_{\gamma}= & \frac{\bar{q} S}{m V} C_{L}^{\delta_{c}}, \\
D_{\gamma}= & d_{\gamma, \alpha_{f}}+d_{\gamma, \delta}+d_{\gamma}, \\
d_{\gamma, \alpha_{f}}= & \frac{\bar{q} S}{m V}\left[\left(C_{T, \Phi}^{3} \alpha^{3}+C_{T, \Phi}^{2} \alpha^{2}+C_{T, \Phi}^{1} \alpha\right) \Phi\right. \\
& +\left(C_{T}^{3} \alpha^{3}+C_{T}^{2} \alpha^{2}+C_{T}^{1} \alpha\right) \\
& -\left(C_{T, \Phi}^{3} \alpha_{f}^{3}+C_{T, \Phi}^{2} \alpha_{f}^{2}+C_{T, \Phi}^{1} \alpha_{f}\right) \Phi \\
& \left.-\left(C_{T}^{3} \alpha_{f}^{3}+C_{T}^{2} \alpha_{f}^{2}+C_{T}^{1} \alpha_{f}\right)\right] \sin \alpha, \\
& \bar{q} S \\
d_{\gamma, \delta}= & \left(C_{L}^{\delta_{e}} \delta_{e}+C_{L}^{\delta_{c}} \Delta \delta_{c}\right) .
\end{aligned}
$$

Considering the real small $\alpha$, the approximate residual $d_{\gamma, \alpha_{f}}$ is assumed to be bounded. Meanwhile, the unknown disturbance introduced by the above control allocation $d_{\gamma, \delta}$ is bounded considering the amplitude and rate constrained actuators. Thus, the boundedness of the disturbance term $D_{\gamma}$ is guaranteed.

Furthermore, similar to [4], the virtual AoA control is designed as

$$
\begin{gathered}
\alpha_{d}=\alpha_{f}-e_{\gamma}, \\
\alpha-\alpha_{f}=\alpha-\alpha_{d}-e_{\gamma}=e_{\alpha}-e_{\gamma} .
\end{gathered}
$$

Then, $\dot{e}_{\gamma}$ can be rewritten as

$$
\dot{e}_{\gamma}=R_{\Gamma}\left(e_{\alpha}-e_{\gamma}\right)+H_{\gamma}+G_{\gamma} \delta_{c}^{0}+D_{\gamma}-\dot{\gamma}_{\text {ref }} .
$$

Similarly, the following NDO for $D_{\gamma}$ is constructed.

$$
\left\{\begin{array}{l}
\dot{p}_{\gamma}=-l_{\gamma}\left[\lambda_{\gamma}(\gamma)+p_{\gamma}+R_{\Gamma}\left(e_{\alpha}-e_{\gamma}\right)+H_{\gamma}+G_{\gamma} \delta_{c}^{0}\right] \\
l_{\gamma}=\frac{\partial \lambda_{\gamma}(\gamma)}{\partial \gamma} \\
\widehat{D}_{\gamma}=p_{\gamma}+\lambda_{\gamma}(\gamma) .
\end{array}\right.
$$

Defining estimation error as $\tilde{D}_{\gamma}=D_{\gamma}-\widehat{D}_{\gamma}$, and

$$
\dot{\tilde{D}}_{\gamma}=-l_{\gamma} \tilde{D}_{\gamma}+\dot{D}_{\gamma} \text {. }
$$

Further noting the bounded $d_{\gamma, \alpha_{f}}, d_{\gamma, \delta}$, and $d_{\gamma}$, the boundedness of $D_{\gamma}$ is guaranteed. From Lemma $3, \tilde{D}_{\gamma}$ is bounded for the fact that $\dot{D}_{\gamma}$ is bounded, i.e., $\left|\tilde{D}_{\gamma}\right| \leq \Theta_{\gamma}$ with $\Theta_{\gamma}>0$.

We design the control law to regulate the $\dot{e}_{\gamma}$ system as

$$
\delta_{c}^{0}=\frac{1}{G_{\gamma}}\left[-k_{\gamma} e_{\gamma}-R_{\Gamma} e_{\alpha}-H_{\gamma}+\dot{\gamma}_{\mathrm{ref}}-D_{\gamma}-\widehat{\Theta}_{\gamma} \tanh \left(\frac{c e_{\gamma}}{\varepsilon_{\gamma}}\right)\right],
$$

where $\widehat{\Theta}_{\gamma}$ is governed by the adaptive law

$$
\dot{\widehat{\Theta}}_{\gamma}=\sigma_{\gamma}\left[-k_{\gamma}^{\prime} \widehat{\Theta}_{\gamma}+e_{\gamma} \tanh \left(\frac{c e_{\gamma}}{\varepsilon_{\gamma}}\right)\right],
$$

with $\sigma_{\gamma}>0, \varepsilon_{\gamma}>0$, and $k_{\gamma}^{\prime}>0$.

3.2.2. Design for $(\alpha, Q)$-Dynamics. For the altitude subsystem, the AoA tracking error dynamics have the relationship

$$
\dot{e}_{\alpha}=\dot{\alpha}-\dot{\alpha}_{d}=\dot{\alpha}-\dot{\alpha}_{f}+\dot{e}_{\gamma}=Q-\dot{\gamma}_{\text {ref }}-\dot{\alpha}_{f} .
$$

Define $e_{Q}=Q-Q_{d}$ with $Q_{d}=-k_{\alpha} e_{\alpha}+\dot{\gamma}_{\text {ref }}+\dot{\alpha}_{f}$, and $\dot{e}_{\alpha}$ can be rewritten as

$$
\begin{gathered}
\dot{e}_{\alpha}=-k_{\alpha} e_{\alpha}+e_{Q}, \\
\dot{Q}_{d}=k_{\alpha}^{2} e_{\alpha}-k_{\alpha} e_{Q}+\ddot{\gamma}_{\mathrm{ref}}+\ddot{\alpha}_{f} .
\end{gathered}
$$

Then, the PR dynamics is formulated as

$$
\dot{Q}=H_{Q}+G_{Q} \delta_{e}^{0}+D_{Q}
$$

where

$$
\begin{aligned}
H_{Q}= & \frac{z_{T} \bar{q} S}{I_{y y}}\left[\left(C_{T, \Phi}^{3} \alpha^{3}+C_{T, \Phi}^{2} \alpha^{2}+C_{T, \Phi}^{1} \alpha+C_{T, \Phi}^{0}\right) \Phi\right. \\
& \left.+\left(C_{T}^{3} \alpha^{3}+C_{T}^{2} \alpha^{2}+C_{T}^{1} \alpha+C_{T}^{0}\right)\right], \\
G_{Q}= & \frac{\bar{q} \bar{c} S}{I_{y y}} C_{M}^{\delta_{e}}, \\
D_{Q}= & d_{Q}+d_{Q, \delta}+\sum_{i=1}^{2} \frac{\psi_{i} \ddot{\eta}_{i}}{I_{y y}}, \\
d_{Q, \delta}= & \frac{\bar{q} \bar{c} S}{I_{y y}}\left(C_{M}^{\delta_{c}} \delta_{c}+C_{M}^{\delta_{e}} \Delta \delta_{e}\right) .
\end{aligned}
$$

Following a similar analysis to previous steps, the boundedness of $D_{Q}$ is also guaranteed. Thus, the PR tracking error dynamics is formulated as

$$
\dot{e}_{Q}=H_{Q}+G_{Q} \delta_{e}^{0}+D_{Q}-k_{\alpha}^{2} e_{\alpha}-\ddot{\gamma}_{\text {ref }}-\ddot{\alpha}_{f} .
$$


Then, NDO for $D_{Q}$ is constructed as

$$
\left\{\begin{array}{l}
\dot{p}_{Q}=-l_{Q}\left[\lambda_{Q}(Q)+p_{Q}+H_{Q}+G_{Q} \delta_{e}^{0}\right] \\
l_{Q}=\frac{\partial \lambda_{Q}(Q)}{\partial Q} \\
\widehat{D}_{Q}=p_{Q}+\lambda_{Q}(Q) .
\end{array}\right.
$$

Defining estimation error as $\tilde{D}_{Q}=D_{Q}-\widehat{D}_{Q}$, it has

$$
\dot{\tilde{D}}_{Q}=-l_{Q} \tilde{D}_{Q}+\dot{D}_{Q} .
$$

$\sum_{i=1}^{2}\left(\psi_{i} \ddot{\eta}_{i} / I_{y y}\right)$ is bounded according to the corresponding analysis in $[25,26]$. Considering the bounded $\dot{d}_{Q}, \dot{d}_{Q, \delta}$, and $\sum_{i=1}^{2}\left(\psi_{i} \ddot{\eta}_{i} / I_{y y}\right)$-differentiator, $\dot{D}_{Q}$ is bounded. Similarly, $\tilde{D}_{Q}$ is bounded for the fact that $\dot{D}_{Q}$ is bounded, i.e., $\left|\tilde{D}_{Q}\right| \leq \Theta_{Q}$ with $\Theta_{Q}>0$.

We design the control law to regulate the $\dot{e}_{Q}$ system as

$\delta_{e}^{0}=\frac{1}{G_{Q}}\left[-k_{Q} e_{Q}+k_{\alpha} e_{\alpha}^{2}-e_{\alpha}-H_{Q}+\ddot{\gamma}_{\text {ref }}+\ddot{\alpha}_{f}-\widehat{D}_{Q}-\widehat{\Theta}_{Q} \tanh \left(\frac{c e_{Q}}{\varepsilon_{Q}}\right)\right]$,

where $\widehat{\Theta}_{Q}$ is governed by the adaptive law

$$
\dot{\widehat{\Theta}}_{Q}=\sigma_{Q}\left[-k_{Q}^{\prime} \widehat{\Theta}_{Q}-e_{Q} \tanh \left(\frac{c e_{Q}}{\varepsilon_{Q}}\right)\right] \text {, }
$$

with $\sigma_{Q}>0, \varepsilon_{Q}>0$, and $k_{Q}^{\prime}>0$.

3.2.3. Control Allocation Design for CDA-EDA. In this subsection, a control allocation algorithm on CDA and EDA is integrated to the proposed control considering the hard magnitude and rate constraints of canard and elevator deflections.

Considering the components $M$ in (10), we briefly define

$$
\begin{gathered}
u_{M}^{\mathrm{com}}=C_{M}^{\delta_{c}} \delta_{c}^{\mathrm{com}}+C_{M}^{\delta_{e}} \delta_{e}^{\mathrm{com}}, \\
u_{M}^{\text {des }}=C_{M}^{\delta_{c}} \delta_{c}^{0}+C_{M}^{\delta_{e}} \delta_{e}^{0},
\end{gathered}
$$

where $u_{M}^{\text {com }}$ denotes the commands generated by control allocation; $u_{M}^{\text {des }}$ presents the desired values.

Meanwhile, assume the future control sequence with $z$ prediction intervals at the current interval $k$ as

$$
\Xi_{k}=\left[\begin{array}{llll}
\delta_{c}^{\mathrm{com}}(k \mid k) & \delta_{c}^{\mathrm{com}}(k+1 \mid k) & \cdots & \delta_{c}^{\mathrm{com}}(k+z-1 \mid k) \\
\delta_{e}^{\mathrm{com}}(k \mid k) & \delta_{e}^{\mathrm{com}}(k+1 \mid k) & \cdots & \delta_{e}^{\mathrm{com}}(k+z-1 \mid k)
\end{array}\right]^{T} .
$$

As the flight control system is a sampled-data system, the magnitude and rate can establish the following simple firstorder approximation:

$$
\dot{\delta} \approx \frac{\delta(t)-\delta\left(t-t_{s}\right)}{t_{s}}
$$

where $t_{s}$ is the control interval and $\delta\left(t-t_{s}\right)$ is the CDA/EDA command generated in the last control interval; the rate constraint can be absorbed into the magnitude constraint:

$$
\delta\left(t-t_{s}\right)+\mathscr{R}_{L} t_{s} \leq \delta(t) \leq \delta\left(t-t_{s}\right)+\mathscr{R}_{U} t_{s} .
$$

And new constraints can be defined as

$$
\begin{gathered}
\mathscr{M}_{\max }=\max \left\{\mathscr{M}_{U}, \delta\left(t-t_{s}\right)+\mathscr{R}_{U} t_{s}\right\}, \\
\mathscr{M}_{\text {min }}=\min \left\{\mathscr{M}_{L}, \delta\left(t-t_{s}\right)+\mathscr{R}_{L} t_{s}\right\} .
\end{gathered}
$$

Therefore, the optimization problem of the above control allocation can be expressed in the following form:

$$
\begin{array}{cc}
\min _{\Xi_{k}} & J\left(\Xi_{k}\right) \\
\text { subject to } & \mathscr{M}_{\text {min }} \leq \delta \leq \mathscr{M}_{\text {max }}
\end{array}
$$

with

$$
J\left(\Xi_{k}\right)=J_{\Delta_{u}}\left(\Xi_{k}\right)+J_{\Delta_{\delta}}\left(\Xi_{k}\right),
$$

$$
J_{\Delta_{u}}\left(\Xi_{k}\right)=\sum_{j=1}^{z}\left\{w_{j}^{u}\left[u_{M}^{\operatorname{com}}(k+j \mid k)-u_{M}^{\operatorname{des}}(k+j \mid k)\right]\right\}^{2},
$$

$$
\begin{aligned}
J_{\Delta_{\delta}}\left(\Xi_{k}\right)= & \sum_{j=1}^{z}\left\{w_{j}^{c}\left[u_{c}^{\mathrm{com}}(k+j \mid k)-u_{c}^{\mathrm{des}}(k+j-1 \mid k)\right]\right\}^{2} \\
& +\sum_{j=1}^{z}\left\{w_{j}^{e}\left[u_{e}^{\mathrm{com}}(k+j \mid k)-u_{e}^{\mathrm{des}}(k+j-1 \mid k)\right]\right\}^{2},
\end{aligned}
$$

where $w_{j}^{*}$ is the tuning weight matrices for the $j$ th step.

Remark 1. The cost function in (52) is set for two main practical purposes. One goal reflected in $J_{\Delta_{u}}\left(\Xi_{k}\right)$ is to pursue approximating precision, and the other goal reflected in $J_{\Delta_{\delta}}$ $\left(\Xi_{k}\right)$ is to maximize energy savings.

\section{Stability Analysis}

In this section, stability and performance properties of the closed-loop systems are summarised. 
Consider the Lyapunov function $\mathscr{L}=\mathscr{L}_{V}+\mathscr{L}_{h, \gamma}+\mathscr{L}_{\alpha, Q}$, where

$$
\begin{gathered}
\mathscr{L}_{V}=\frac{1}{2}\left(e_{V}^{2}+\frac{1}{\sigma_{V}} \tilde{\Theta}_{V}^{2}\right), \\
\mathscr{L}_{h, \gamma}=\frac{1}{2}\left(e_{\gamma}^{2}+\frac{1}{\sigma_{\gamma}} \tilde{\Theta}_{\gamma}^{2}\right), \\
\mathscr{L}_{\alpha, Q}=\frac{1}{2}\left(e_{\alpha}^{2}+e_{Q}^{2}+\frac{1}{\sigma_{Q}} \tilde{\Theta}_{Q}^{2}\right) .
\end{gathered}
$$

Utilizing (23), (27) together with Lemma 1 and applying inequalities: $2 \tilde{\Theta}_{V} \widehat{\Theta}_{V} \leq \Theta_{V}^{2}-\tilde{\Theta}_{V}^{2}, \dot{\mathscr{L}}_{V}$ satisfies

$\dot{\mathscr{L}}_{V}=e_{V} \dot{e}_{V}+\frac{1}{\sigma_{V}} \tilde{\Theta}_{V} \dot{\tilde{\Theta}}_{V} \leq-k_{V} e_{V}^{2}-\frac{k_{V}^{\prime}}{2} \tilde{\Theta}_{V}^{2}+\left(\frac{k_{V}^{\prime}}{2} \Theta_{V}+\varepsilon_{V}\right) \Theta_{V}$

Similarly, $\mathscr{L}_{h, \gamma}$ and $\mathscr{L}_{\alpha, Q}$ can be calculated from (32), (35), (36) and (41), (44), (45), respectively.

$$
\begin{gathered}
\dot{\mathscr{L}}_{h, \gamma}=e_{\gamma} \dot{e}_{\gamma}+\frac{1}{\sigma_{\gamma}} \tilde{\Theta}_{\gamma} \dot{\tilde{\Theta}}_{\gamma} \leq-\left(k_{\gamma}+R_{\Gamma}\right) e_{\gamma}^{2}-\frac{k_{\gamma}^{\prime}}{2} \tilde{\Theta}_{\gamma}^{2}+\left(\frac{k_{\gamma}^{\prime}}{2} \Theta_{\gamma}+\varepsilon_{\gamma}\right) \Theta_{\gamma}, \\
\dot{\mathscr{L}}_{\alpha, Q}=e_{\alpha} \dot{e}_{\alpha}+e_{Q} \dot{e}_{Q}+\frac{1}{\sigma_{Q}} \tilde{\Theta}_{Q} \dot{\tilde{\Theta}}_{Q} \leq-k_{\alpha} e_{\alpha}^{2}-\left(k_{Q}-k_{\alpha}\right) e_{Q}^{2} \\
-\frac{k_{Q}^{\prime}}{2} \tilde{\Theta}_{Q}^{2}+\left(\frac{k_{Q}^{\prime}}{2} \Theta_{Q}+\varepsilon_{Q}\right) \Theta_{Q} .
\end{gathered}
$$

By adding inequalities (56)-(58), we obtain

$$
\begin{aligned}
\dot{\mathscr{L}} \leq & -k_{V} e_{V}^{2}-\left(k_{\gamma}+R_{\Gamma}\right) e_{\gamma}^{2}-k_{\alpha} e_{\alpha}^{2}-\left(k_{Q}-k_{\alpha}\right) e_{Q}^{2}-\frac{k_{V}^{\prime}}{2} \tilde{\Theta}_{V}^{2} \\
& -\frac{k_{\gamma}^{\prime}}{2} \tilde{\Theta}_{\gamma}^{2}-\frac{k_{Q}^{\prime}}{2} \tilde{\Theta}_{Q}^{2}+\left(\frac{k_{V}^{\prime}}{2} \Theta_{V}+\varepsilon_{V}\right) \Theta_{V}+\left(\frac{k_{\gamma}^{\prime}}{2} \Theta_{\gamma}+\varepsilon_{\gamma}\right) \Theta_{\gamma} \\
& +\left(\frac{k_{Q}^{\prime}}{2} \Theta_{Q}+\varepsilon_{Q}\right) \Theta_{Q} \leq-a_{\mathscr{L}} \mathscr{L}+b_{\mathscr{L}},
\end{aligned}
$$

where $k_{Q}>k_{\alpha}, k_{\gamma}>-R_{\Gamma}$, and $a_{\mathscr{L}}, b_{\mathscr{L}} \in \mathbb{R}_{+}$are constants expressed as

$$
\begin{aligned}
& a_{\mathscr{L}}=\min \left\{2 k_{V}, 2\left(k_{\gamma}+R_{\Gamma}\right), 2 k_{\alpha}, 2\left(k_{Q}-k_{\alpha}\right), k_{V}^{\prime} \sigma_{V}, k_{\gamma}^{\prime} \sigma_{\gamma}, k_{Q}^{\prime} \sigma_{Q}\right\}, \\
& b_{\mathscr{L}}=\left(\frac{k_{V}^{\prime}}{2} \Theta_{V}+\varepsilon_{V}\right) \Theta_{V}+\left(\frac{k_{\gamma}^{\prime}}{2} \Theta_{\gamma}+\varepsilon_{\gamma}\right) \Theta_{\gamma}+\left(\frac{k_{V}^{\prime}}{2} \Theta_{Q}+\varepsilon_{Q}\right) \Theta_{Q} .
\end{aligned}
$$

Obviously, $\dot{\mathscr{L}}<0$ provided that $\mathscr{L}>b_{\mathscr{L}} / a_{\mathscr{L}}$. Therefore, all signals involved in the Lyapunov function and utilized in the design are uniformly ultimately bounded.

Applying Lemma 2 to (59), it is concluded that the boundedness of the related variables and signals in the proposed control can be ensured. Meanwhile, the tracking error ex with $x=V, h$ retains the uniformly ultimate boundedness property:

$$
\max \left\{\left|e_{V}(t)\right|,\left|e_{h}(t)\right|\right\} \leq \sqrt{2 \frac{b_{\mathscr{L}}}{a_{\mathscr{L}}}+\left[\mathscr{L}(0)-\frac{b_{\mathscr{L}}}{a_{\mathscr{L}}}\right] e^{-a_{\mathscr{L}} t}},
$$

with

$$
\lim _{t \rightarrow \infty} \max \left\{\left|e_{V}(t)\right|,\left|e_{h}(t)\right|\right\} \leq \sqrt{2 \frac{b_{\mathscr{L}}}{a_{\mathscr{L}}}} .
$$

\section{Simulation Results}

This section implements a simulation study to verify the proposed controller. In the simulating model, the nominal parameter values of the AHV model are selected according to [1]. For comparison, a slightly modified version of the adaptive fault-tolerant control presented in [9] is applied to the same scenario (denoted as "compared control" for convenience).

The simulated AHV is initially cruising at $V(0)=7846$ $\mathrm{ft} / \mathrm{s}, \quad h(0)=85000 \mathrm{ft}, \quad \gamma(0)=0 \mathrm{rad}, \alpha(0)=2.6 \mathrm{deg}, \quad \eta_{1}(0)=$ $0.92 \mathrm{ft} \cdot \sqrt{\text { slugs }} / \mathrm{ft}$, and $\eta_{2}(0)=0.61 \mathrm{ft} \cdot \sqrt{\text { slugs }} / \mathrm{ft}$ and plans to implement a maneuver in the next $600 \mathrm{~s}$. $\Phi_{\text {cooling }}=0.01$ and $\Phi_{\text {choking }}$ is selected according to [12]; $\mathscr{M}_{L}=-20 \mathrm{deg}$, $\mathscr{M}_{U}=20 \mathrm{deg}, \mathscr{R}_{L}=-60 \mathrm{deg} / \mathrm{s}$, and $\mathscr{R}_{U}=60 \mathrm{deg} / \mathrm{s}$; the simulating control interval is set as $t_{s}=10 \mathrm{~ms}$.

Velocity and altitude references are generated by passing step command signals:

$$
\begin{aligned}
& V_{c}(t)=\left\{\begin{array}{l}
7,846, t=0 \mathrm{~s}, \\
9,846, t \in(0 \mathrm{~s}, 300 \mathrm{~s}], \\
9,000, t \in(300 \mathrm{~s}, 600 \mathrm{~s}],
\end{array}\right. \\
& h_{c}(t)=\left\{\begin{array}{l}
85,000, t=0 \mathrm{~s}, \\
110,000, t \in(0 \mathrm{~s}, 300 \mathrm{~s}], \\
95,000, t \in(300 \mathrm{~s}, 600 \mathrm{~s}],
\end{array}\right.
\end{aligned}
$$

through a second-order filter [4] with the damping ratio of 0.9 and the natural frequency of $0.03 \mathrm{rad} / \mathrm{s}$.

The control parameters are set as follows. In the velocity loop, $k_{V}=5, k_{V}^{\prime}=10, \sigma_{V}=0.1, \varepsilon_{V}=1$, and $l_{V}=5$. In the altitude loop, $k_{\gamma}=20, k_{\gamma}^{\prime}=1, \sigma_{\gamma}=0.5, \varepsilon_{\gamma}=5, l_{\gamma}=10 ; k_{\alpha}=10$, $k_{\mathrm{Q}}=40, k_{\mathrm{Q}}^{\prime}=0.3, \sigma_{\mathrm{Q}}=0.8, \varepsilon_{\mathrm{Q}}=10$, and $l_{\mathrm{Q}}=20$.

The main simulating results are given in Figures 1-9. Figures 1 and 2 show the output tracking results during the ascending maneuver and the descending maneuver, from which we see that the proposed control performs better, 


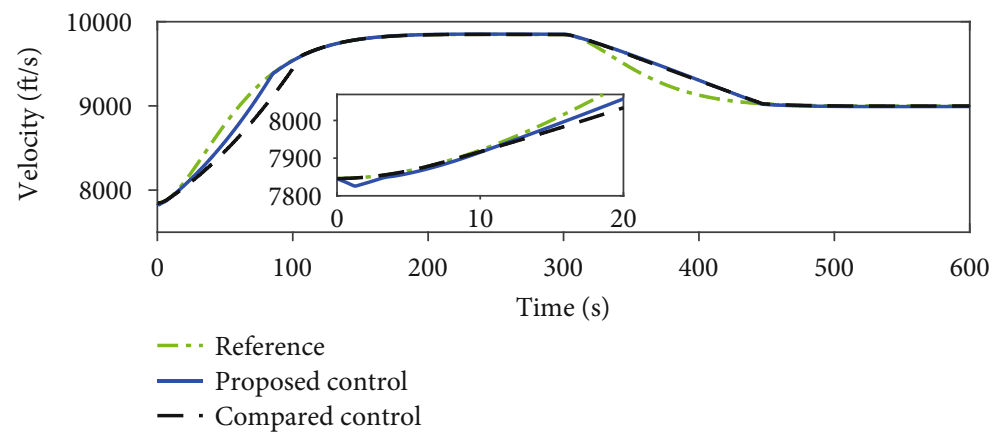

(a)

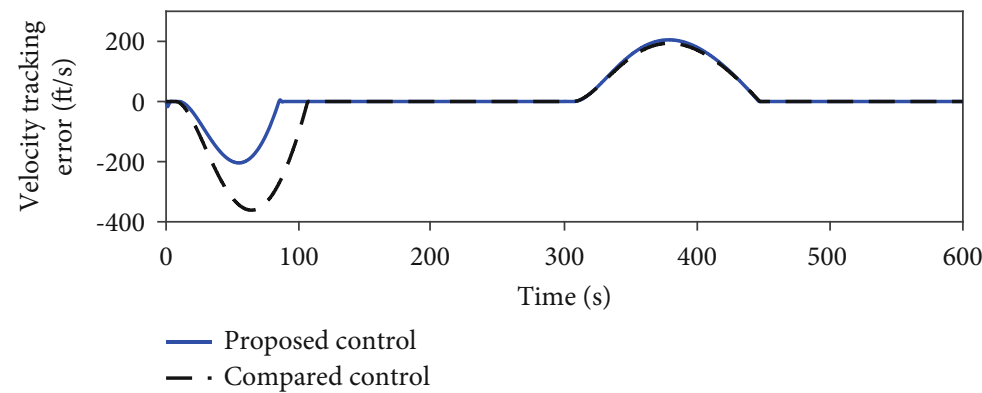

(b)

FIGURE 1: Velocity tracking results in the comparison simulation: (a) velocity tracking; (b) velocity tracking error.

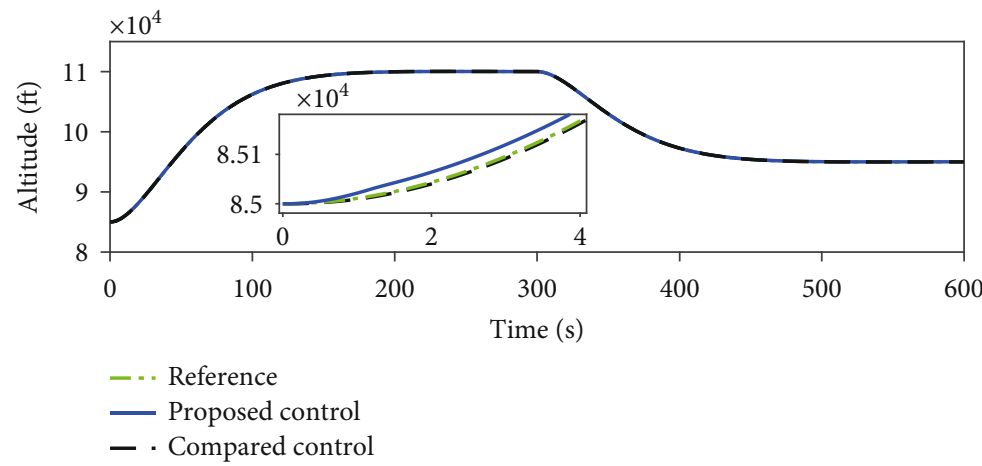

(a)

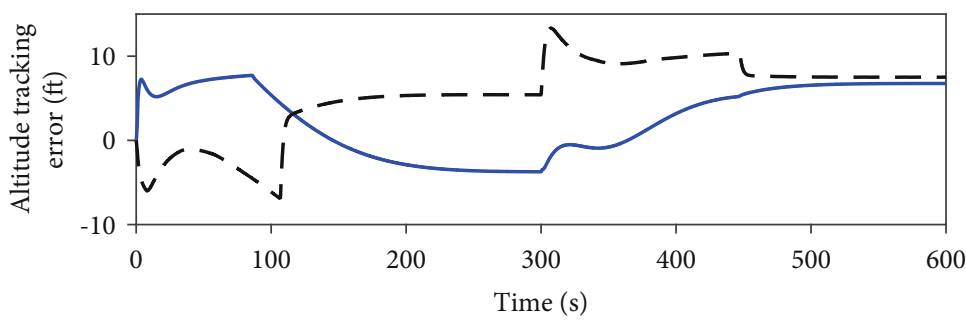

- Proposed control

- Compared control

(b)

FIGURE 2: Altitude tracking results in the comparison simulation: (a) altitude tracking; (b) altitude tracking error. 


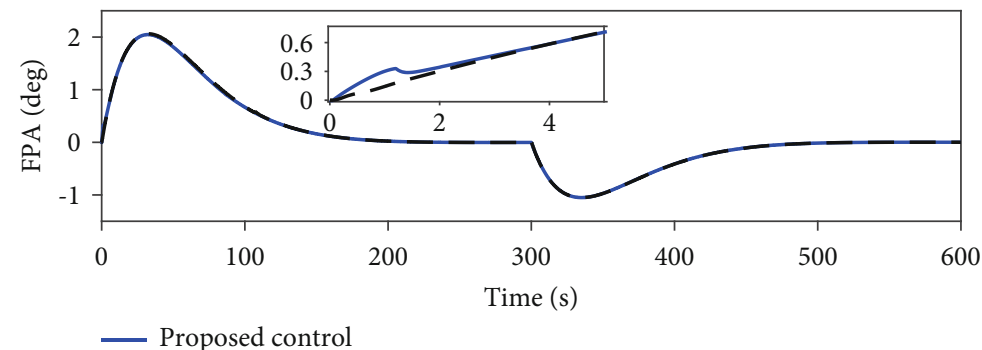

(a)

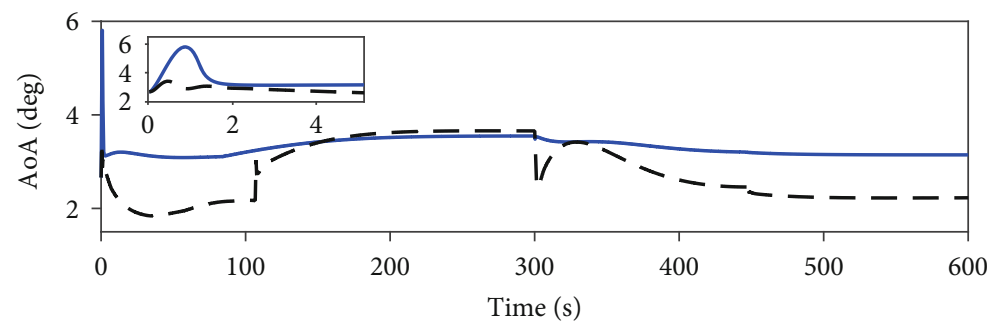

- Proposed control

- Compared control

(b)

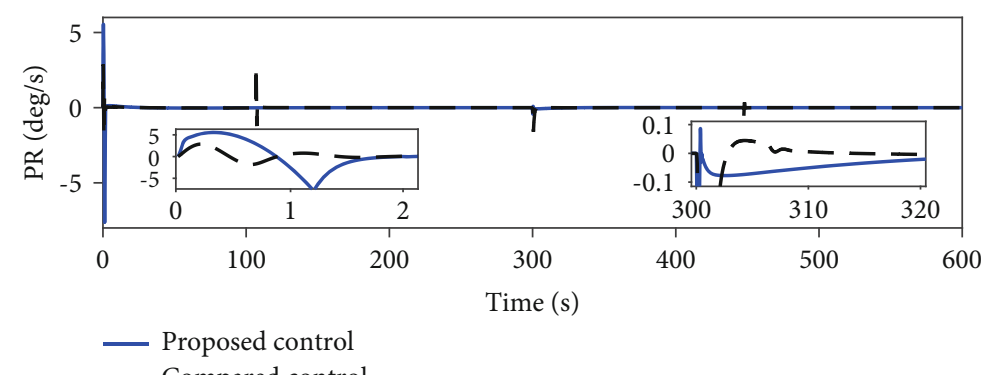

(c)

Figure 3: Flight attitudes in the comparison simulation: (a) flight path angle; (b) angle of attack; (c) pitch rate.

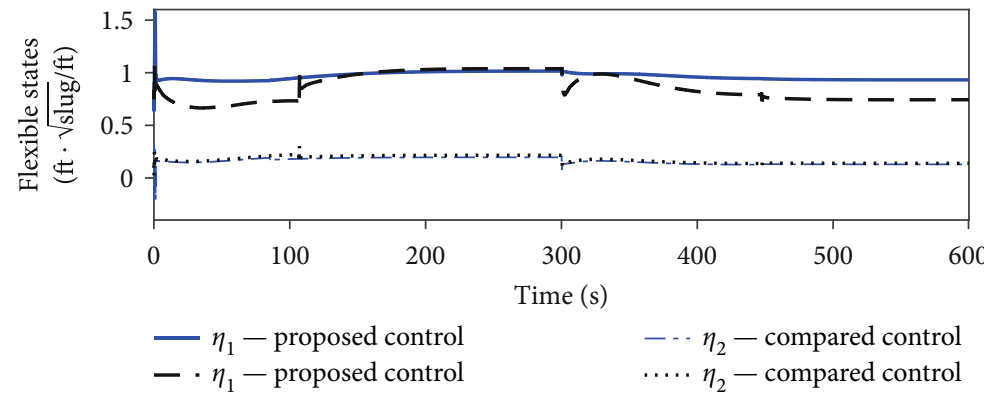

FIgURE 4: Flexible states in the comparison simulation.

though both controls achieve the desired conditions. Time histories of flight attitudes including FPA, AoA, and PR are given in Figure 3. It is seen from Figure 1 that the desired velocity reference cannot be tracked because of the FER saturation property, also see Figure 5. In this situation, the employed auxiliary system generates a modified reference, which is less aggressive and can be well tracked within the ability of scramjet. Figure 4 shows the flexible states in the comparison simulation. Figures 5-7 indicate the control input signals of FER, CDA, and EDA. It is seen from Figures 6 and 7 that both the magnitude constraints and rate constraints of CDA/EDA are well constrained within prescribed bound under the proposed control, which performs better than the compared control. Figure 8 shows the results 


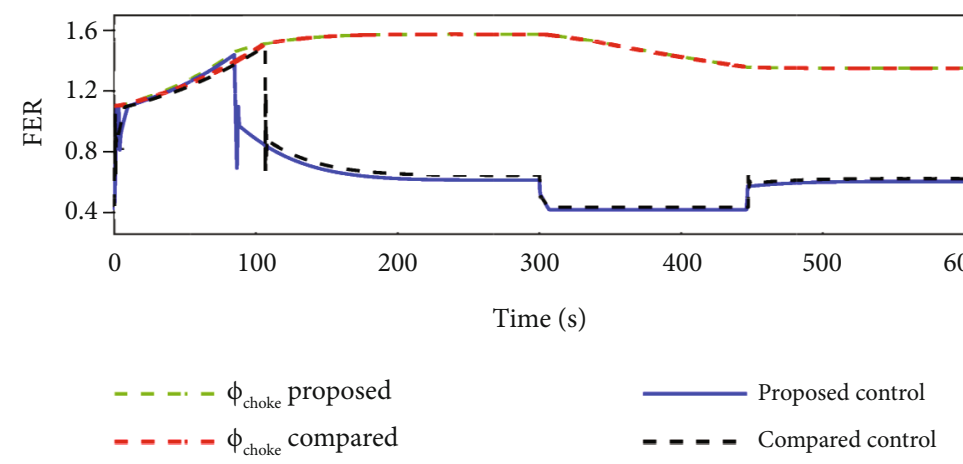

(a)

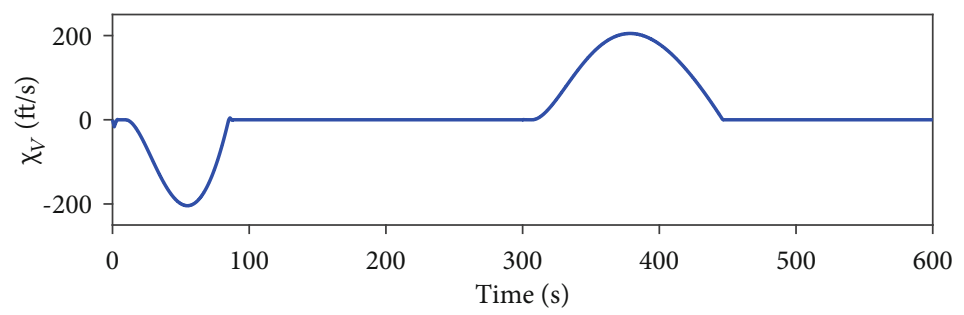

(b)

FIGURE 5: FER and state of the auxiliary system $\chi_{V}$ : (a) FER; (b) state of the auxiliary system.



- Proposed control

- Compared control

(a)

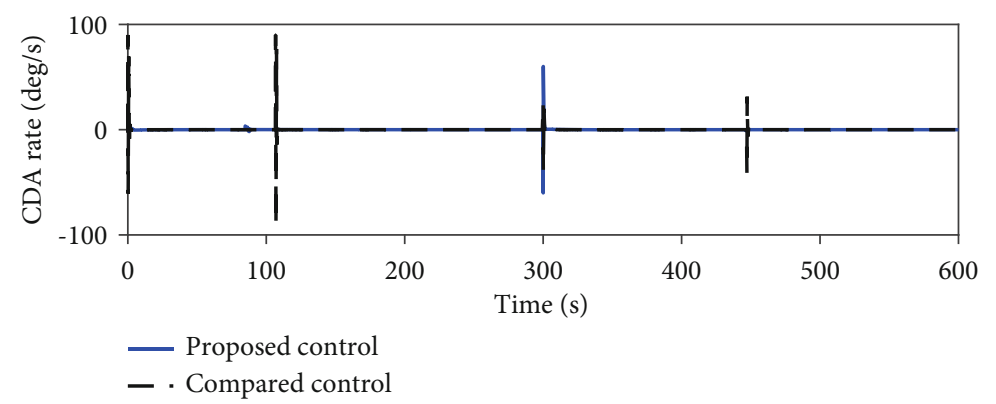

(b)

FIGURE 6: CDA and CDA rate in the comparison simulation: (a) canard deflection angle; (b) canard deflection angle rate. 


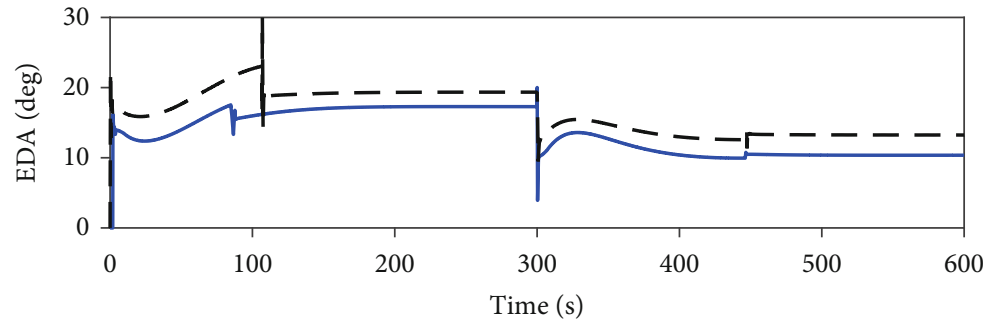

- Proposed control

- Compared control

(a)

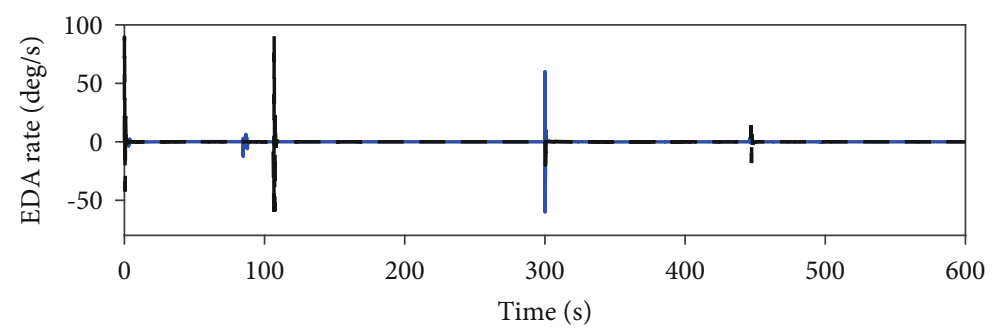

- Proposed control

- Compared control

(b)

FIGURE 7: EDA and EDA rate in the comparison simulation: (a) elevator deflection angle; (b) elevator deflection angle rate.

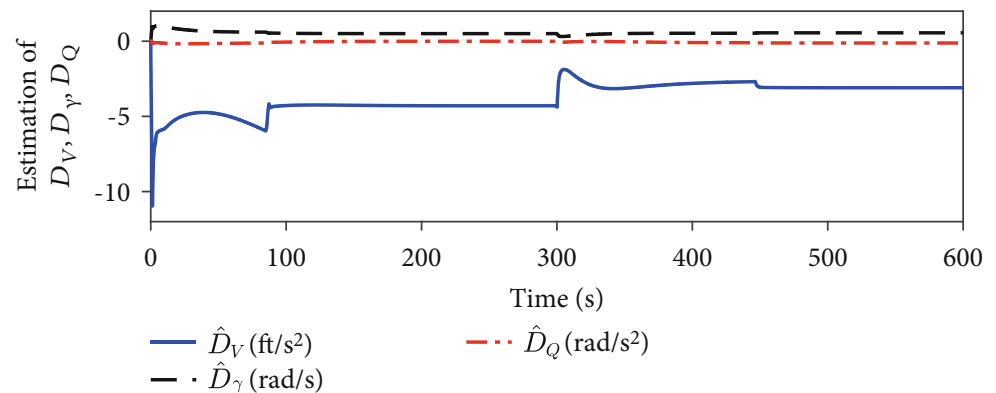

FIGURE 8: Results of lumped disturbance estimation.

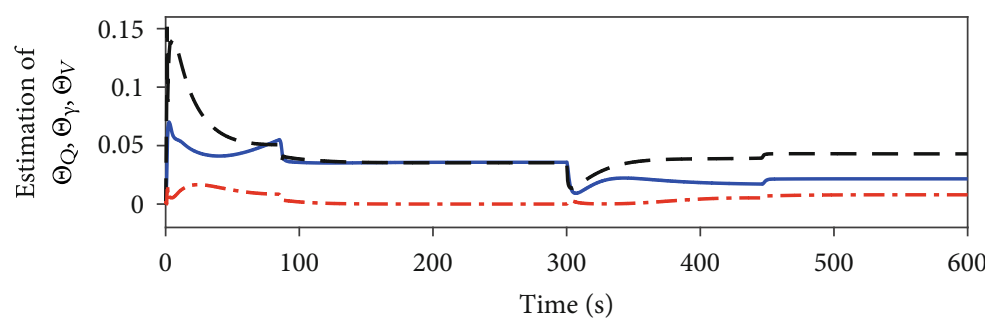

$-\hat{\Theta}_{V}$

$-\cdot \hat{\Theta}_{\gamma}$

$-\cdots \hat{\Theta}_{\mathrm{Q}}$

FIgURE 9: Estimation errors $\widehat{\Theta}_{V}, \widehat{\Theta}_{\gamma}$, and $\widehat{\Theta}_{Q}$. 
of lumped disturbance estimation from NDOs. $\widehat{\Theta}_{V}, \widehat{\Theta}_{\gamma}$, and $\widehat{\Theta}_{Q}$ estimated by the adaptive laws are shown in Figure 9.

\section{Conclusions}

In this paper, a disturbance observer-based adaptive control for a hypersonic vehicle has been addressed to obtain the capability of handling external disturbances and actuator constraints. Fast disturbance estimate has been addressed via NDO and adaptive control compensation in each subsystem. An auxiliary system has been employed subject to the scramjet input saturation. Meanwhile, there is an effective mechanism of dealing with the magnitude/rate constraints and dynamics of aerodynamic control surfaces by introducing a control allocation module. Simulations have been provided to verify the effectiveness of the proposed control.

\section{Data Availability}

The data used to support the findings of this study are available from the corresponding author upon request.

\section{Conflicts of Interest}

The authors declare that there is no conflict of interest regarding the publication of this paper.

\section{Acknowledgments}

This work was supported by the National Natural Science Foundation of China (grant number 61304108).

\section{References}

[1] J. T. Parker, A. Serrani, S. Yurkovich, M. A. Bolender, and D. B. Doman, "Control-oriented modeling of an air-breathing hypersonic vehicle," Journal of Guidance, Control, and Dynamics, vol. 30, no. 3, pp. 856-869, 2007.

[2] B. Xu and Z. Shi, "An overview on flight dynamics and control approaches for hypersonic vehicles," Science China Information Sciences, vol. 58, no. 7, pp. 1-19, 2015.

[3] H. Xu, M. D. Mirmirani, and P. A. Ioannou, "Adaptive sliding mode control design for a hypersonic flight vehicle," Journal of Guidance, Control, and Dynamics, vol. 27, no. 5, pp. 829-838, 2004.

[4] L. Fiorentini, A. Serrani, M. A. Bolender, and D. B. Doman, "Nonlinear robust adaptive control of flexible air-breathing hypersonic vehicles," Journal of Guidance, Control, and Dynamics, vol. 32, no. 2, pp. 402-417, 2009.

[5] H. An, Q. Wu, C. Wang, and X. Cao, "Scramjet operation guaranteed longitudinal control of air-breathing hypersonic vehicles," IEEE/ASME Transactions on Mechatronics, vol. 1, pp. 1-11, 2020.

[6] X. Hu, L. Wu, C. Hu, and H. Gao, "Adaptive sliding mode tracking control for a flexible air-breathing hypersonic vehicle," Journal of the Franklin Institute, vol. 349, no. 2, pp. 559-577, 2012.

[7] L. Fiorentini and A. Serrani, "Adaptive restricted trajectory tracking for a non-minimum phase hypersonic vehicle model," Automatica, vol. 48, no. 7, pp. 1248-1261, 2012.
[8] Z. Yu, Y. Zhang, B. Jiang et al., "Decentralized fractional-order backstepping fault-tolerant control of multi-UAVs against actuator faults and wind effects," Aerospace Science and Technology, vol. 77, pp. 189-205, 2017.

[9] H. An, B. Fidan, J. Liu, C. Wang, and L. Wu, “Adaptive faulttolerant control of air-breathing hypersonic vehicles robust to input nonlinearities," International Journal of Control, vol. 92, no. 5, pp. 1044-1060, 2017.

[10] X. Bu, X. Wu, D. Wei, and J. Huang, "Neural approximationbased robust adaptive control of flexible air-breathing hypersonic vehicles with parametric uncertainties and control input constraints," Information Sciences, vol. 346-347, pp. 29-43, 2016.

[11] Z. Yu, Z. Liu, Y. Zhang, Y. Qu, and C. Su, "Distributed finitetime fault-tolerant containment control for multiple unmanned aerial vehicles," IEEE Transactions on Neural Networks and Learning Systems, vol. 31, no. 6, pp. 2077-2091, 2020.

[12] A. Zinnecker, A. Serrani, M. Bolender, and D. Doman, "Combined reference governor and anti-windup design for constrained hypersonic vehicles models," in AIAA Guidance, Navigation, and Control Conference, pp. 6283-6283, Chicago, IL, USA, August 2009.

[13] D. Wiese, A. Annaswamy, J. Muse, and M. Bolender, "Adaptive control of a generic hypersonic vehicle," in AIAA Guidance, Navigation, and Control Conference, pp. 4514-4514, Boston, MA, USA, August 2013.

[14] H. An, C. Wang, and B. Fidan, "Sliding mode disturbance observer-enhanced adaptive control for the air-breathing hypersonic flight vehicle," Acta Astronautica, vol. 139, pp. 111-121, 2017.

[15] X. Bu, X. Wu, M. Tian, J. Huang, R. Zhang, and Z. Ma, "Highorder tracking differentiator based adaptive neural control of a flexible air-breathing hypersonic vehicle subject to actuators constraints," ISA Transactions, vol. 58, pp. 237-247, 2015.

[16] H. An, J. Liu, C. Wang, and L. Wu, "Disturbance observerbased antiwindup control for air-breathing hypersonic vehicles," IEEE Transactions on Industrial Electronics, vol. 63, no. 5, pp. 3038-3049, 2016.

[17] X. Bu, D. Wei, and G. He, "A robust constrained control approach for flexible air-breathing hypersonic vehicles," International Journal of Robust and Nonlinear Control, vol. 30, no. 7, pp. 2752-2776, 2020.

[18] T. A. Johansen and T. I. Fossen, "Control allocation-a survey," Automatica, vol. 49, no. 5, pp. 1087-1103, 2013.

[19] W. Qin, B. He, G. Liu, and P. Zhao, "Robust model predictive tracking control of hypersonic vehicles in the presence of actuator constraints and input delays," Journal of the Franklin Institute, vol. 353, no. 17, pp. 4351-4367, 2016.

[20] L. Chen, C. Edwards, H. Alwi, and M. Sato, "Flight evaluation of a sliding mode online control allocation scheme for fault tolerant control," Automatica, vol. 114, article 108829, 2020.

[21] Z.-P. Jiang and L. Praly, "Design of robust adaptive controllers for nonlinear systems with dynamic uncertainties," Automatica, vol. 34, no. 7, pp. 825-840, 1998.

[22] M. Krstic, P. Kokotovic, and I. Kanellakopoulos, Nonlinear and Adaptive Control Design, John Wiley \& Sons, Inc., 1995.

[23] H. An, J. Liu, C. Wang, and L. Wu, "Approximate backstepping fault-tolerant control of the flexible air-breathing hypersonic vehicle," IEEE/ASME Transactions on Mechatronics, vol. 21, no. 3, pp. 1680-1691, 2015. 
[24] J. Dickeson, A. Rodriguez, S. Sridharan et al., "Control relevant modeling, analysis, and design for scramjet-powered hypersonic vehicles," in 16th AIAA/DLR/DGLR International Space Planes and Hypersonic Systems and Technologies Conference, p. 7287, Bremen, Germany, October 2009.

[25] B. Xu, D. Wang, Y. Zhang, and Z. Shi, "DOB-based neural control of flexible hypersonic flight vehicle considering wind effects," IEEE/ASME Transactions on Mechatronics, vol. 64, no. 11, pp. 8676-8685, 2017.

[26] M. A. Bolender and D. B. Doman, "Nonlinear longitudinal dynamical model of an air-breathing hypersonic vehicle," Journal of Spacecraft and Rockets, vol. 44, no. 2, pp. 374-387, 2007. 